\title{
Emergency Decision Support System of Urban Rail Transit Based on Business Process Technology
}

\author{
Lili Chen, Saiqiao Zhang \\ Nari Technology Development Limited Company, Rm 3411, No. 20, Gaoxin Road, Pukou District, \\ Nanjing 210000, China;
}

Keywords: Emergency Decision Support System, Urban Rail Transit, Business Process

\begin{abstract}
This paper describes the decision support system based on business process technology which could apply various data and models to assist decision-makers at each level in achieving effective and scientific decisions in the presence of emergencies in the field of urban rail transit. Combining with the development and actual demand of urban rail transit recently, the paper explains the designed idea of using workflow method in the decision-making process, which could remain flexible in building all kinds of emergency decision-making logic, and help obtain an automatic control of emergency linkage actions. An idea of organization of rail transit command center is given in the paper with the characteristics and structure of emergency decision support system.
\end{abstract}

\section{Introduction}

Urban rail transit in China has been developed into a new stage from individual lines to a network, metro operation has also evolved into a complex network system, and there are large amounts of equipment to be maintained and massive data to be processed. It has prominent significance to research and develop the emergency command center of urban rail transit with independent intellectual property rights. Furthermore, it is adapted to the network operation, emergency processing and decision making needs, which could help raise the level of urban rail transit system, the entire reliable security and the level of urban passenger transportation organization system. Tools and technical supports are provided to emergency preplan and its management, such as emergency disposal preplan, process monitoring and effectiveness evaluation of emergency disposal.

Emergency Decision Support System (EDSS) is an important part of the monitoring system software in rail transit command center. It aims at the semi-structured decision problem in rail transit command center, assisting and guiding the disposal of dispatcher under routine or emergency conditions. It is an intelligent human-machine system for supporting the dispatcher to make decisions. The system can provide the required data, information and background for the scheduling decision makers, help establish and maintain decision-making mode. Also, it is able to offer a variety of alternative solutions. It can provide necessary supports for making decisions correctly and quickly through the analysis, comparison of various options and judgment of human-computer interaction function. In addition, it can be controlled in automatic or semi-automatic mode. In allusion to the peculiarity of crowded population within confined space, which has difficulty evacuating passengers in metro operation system, all sorts of detailed process with possible emergency is established. To integrate the data resources in command center, the decision making process of expert system is displayed in the process of routine or emergency disposal, which could ensure the dispatcher to operate with enough references during the execution of preplans and make the dispatcher's work to be more standardized and efficient.

\section{Software architecture}

Considering that there is a possibility to exist various processing flow in an emergency preplan, design of standard business flow is used in EDSS. B/S architecture is adopted to run the functions on the browser interface to edit and display the preplan, and query historical data using BPMN 
diagram form. Meanwhile, the background software on the server implements the functions like logic calculation, judgment and control. The operator can draw any decision-making process flow chart flexibly through the choice of modules and attachment on the browser. The flow chart, which supports parallel processing of multiple branches and conditional jump, as well as the combination of modules, can run on multiple dispatcher workstations at the same time, and to perform process of different branches is allowed in EDSS system on different operator's workstations, which could handle parallel operations of the internal coordination between multiple scheduling automatically.

The organization of Emergence Command Center (ECC) is set to manage emergency command after the lines of urban rail transit form to a network. The system organization of ECC is shown as below in Fig 1.

The NECS (Network Emergency Command System) of COCC (Coordinate Operation Command Center) has the highest authority of the emergency treatment. Not only the control of internal affairs in rail transit, it's also needed to cooperate with other external departments and agencies. The LECS (Line Emergency Command System) which works on OCC(Operation Coordination Center) report to NECS if unexpected event occurs, thereafter the NECS starts the emergency preplan if critical event occurs, it will notify relevant LECSs, then the LECS in the respective line will perform corresponding emergency preplans. NEMS (Network Emergency Monitoring System) works on NCC (Network control center), and NECS reports implementation steps and results of emergency command function to it.

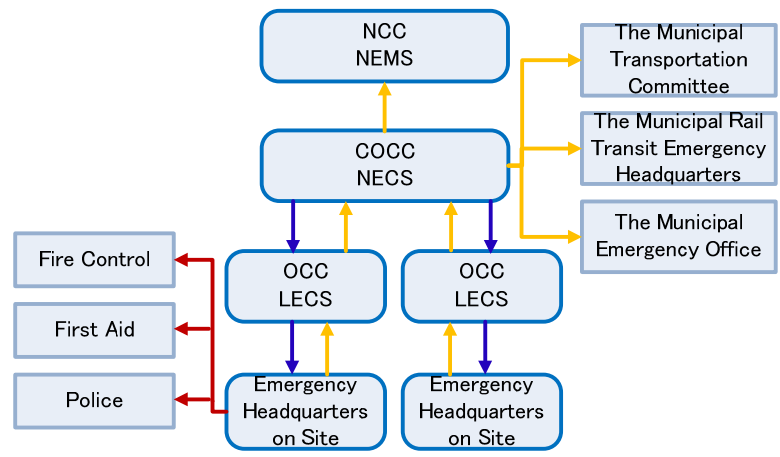

Fig 1 System organization of ECC

Functional modules are loaded automatically when the EDSS tool starts, which could provide users a flexible way to edit graph model of fault diagnosis, emergence preplan. Many functions, for example, topology check, model storage, data management and display of operation results, are integrated in the software, which makes it become a powerful graphical operation platform. Decision-making process in the database is loaded dynamically when the background services program is running. The user interface and the background program coordinate synchronously and interactively, and logic operations of the input points are triggered and real-time values are displayed on the interface of browser.

\section{Software functions}

EDSS system software is used for auxiliary decision-making in rail transit command system to make linkage, sequence control and other emergence response functions, and the BPMN standard is referenced in the software, which could support the editor to draw workflow charts in the form of graphics designer, and the BPMN standard is extended according to the requirement of the rail transit command. Following main functions are included in EDSS: editor of decision-making processes, data processing, fault trigger, operation monitoring, and emergency processing, etc.

Its functions can be grouped into the following categories:

1. Event evaluation and judgment

Based on the decision making process, decision support system collects relevant data and information from each subsystem, captures data of the process states, and monitors the work condition of the integrated system at the same time. The system working status of the equipment could be displayed intuitively and quickly. It calculates the process on time when it is triggered 
provides decision-making reference to monitor automatically in rail transit command center, shares the information resources which could make rail transit to coordinate and cooperate with each other between each device. To achieve the effect of aiding the decision making, it is responsible for the operation of the rail transit on the rails, and collects the normal state of equipment, completes coordination management, prevents the happening of the accident.

\section{Preplan management}

The main goal of emergency preplan management is to speed up the information communication and action response to improve the rationality and scientificity of the decision. A variety of emergency preplans are provided by system according to user's requirements, as well as the preplans for electronic template to provide customers with a custom plan generation and management functions. Data from the subordinate unit can update preplans on time through digital preplan management; superior competent department can retrieve the latest text and other peripheral information of preplans through the plan system. Then the dynamic management of emergency preplans is realized through the interaction between the higher and lower department, the command center can also master major hazards and changes of emergency preplan which exist in each line and each station in a timely manner.

\section{Emergency response command}

If emergency accidents happen at any point in the process of the rail transit operation, it will damage the well-organized operation, and any improper disposal may lead to the losses of people's life and property. So set beforehand all sorts of complex of emergency preplan when event occurs, there would be easy to obtain a closed-loop control to assist emergency response organization and restore operation order as soon as possible, which could ensure operations smoothly and prevent serious accidents to be happened. Query the historical record of the auxiliary decision-making process in the database, and display the result to the interface of historical inquiry.

\section{Preplan exercises and drills}

EDSS has the function of writing and issuing plan of the desktop drill, and manages the processes of training exercises and records data at the same time. The competent department can inspector in case of emergency plan training and drills through the system.

To evaluate plan drilling process, including the adaptability, adequacy, the situation whether the participants, rescue material, and the personal protection are in place, the overall organization and the assignment of responsibility during the rescue, the situation of coordination between departments, then that the evaluation of the overall preplans is concluded finally. Exercise evaluation results can be the input of the preplan in its revision process.

\section{Geographic information services}

Geospatial data and GIS map are used for the operation of emergency command, EDSS provides the function of map plotting, driving route of rescue and then adjusts the drawing function such as automatic search and rescue resources to express the rescue scene and rescue status information.

The GIS function is embodied in the following aspects: disaster simulation, intelligent disposal scheme, evacuation path planning, emergency rescues and 3D data editing management, and so on.

6. Duty and document management

EDSS can be able to achieve duty scheduling of both line and network and make the scheduling management, query information and arrange personnel succession on duty. There are large amounts of documents exist in ECC system which need to manage, for example, preplans, accident reports, duty records, and audio, video, images, log files generating in the process of performing the preplans, and version management is also required by a lot of documents.

\section{Accident report}

The following selective electronic information are used to prepare the emergency assessment report: real-time monitoring video and related images of a line or a station, inner data of the traffic command center, materials of data management system of the auxiliary decision-making, words, forms and image processors, and the reports of individual accidents and full-year track of traffic accidents. 


\section{Implementation scheme}

Background service program of EDSS can scan and calculate the triggered process, treat several key functions of the application logic of command system by a special process.

When the Command system starts, the background server starts as well, it reads the ECC information table in database, generates the tree structure of control flow, and initializes the message bus, loads each process information in real-time database, receives the reconstruct the message in front-end application tool, execution module functions which has four types in the database, i.e. Event, Gateway, Process, Task modules, and execute the triggered control flow.

A state machine is maintained by the background software in system, by which the BPMN workflow logic is implemented to determine the next jump after the execution of current action module. Implementation steps of the system are shown in Fig 2:

1) Load the EDSS trees in the initialization state, and generate relational tables of modules.

2) Maintain the mapping table of triggered events, and determine whether to trigger the corresponding EDSS process tree.

3) Maintain an active module queue by the basic framework of background software, use a state machine to control the node transition, scan activity module queue in idle time, and turn to the next node according to the current module state.

If the initial state of the active module in queue is waiting, then execute the run () function of the module, the module status will be set to running, and if the module has a new thread properties, start a new thread to run the module function. The state of the module will be set to pass or fail at the end, and if the module state is set to pass or fail status, read the next module in queue of current module from the state machine, and remove current module from the heap of active queue.

4) Trigger a calculation in background if various events occur, set the status of current module, and push the next module into heap to be running.

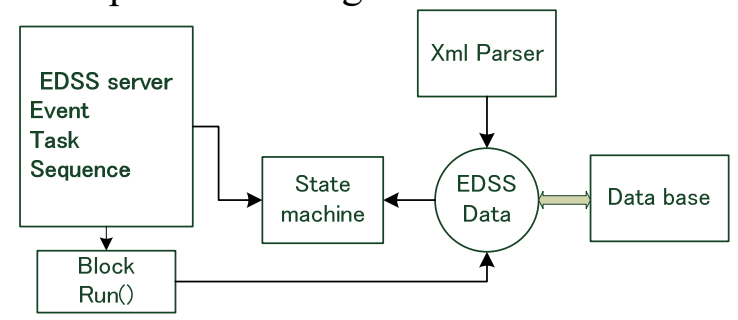

Fig 2 Structure of the background software of EDSS

\section{Summary}

When reviewing the history of the development of urban rail transit at domestic and overseas comprehensively, the emergency management is always the key point of the organization and management of the operation of urban rail traffic. Emergency response requires the integration and assessment of a large set of scientific data from distributed sources to inform actions to reduce the negative influence on the accident. EDSS has been developed so as to allow scientists analyze an accident and communicate recommendations for decision-makers to fulfill this mandate.

Nowadays, the rail transit lines at all levels in a region constitute a network system, and the emergency decision support system has become an indispensable part of the whole system. Considering the aspect of railway traffic control, there are requirements to coordinate operations in an effective manner and share the information resources between each step in urban rail transit operation management process, and ensure that it will proceed normally and everything will be under control if accident happens. To make emergency preplan and implement a risk evaluation and mitigation strategy, it could ensure that rail traffic runs smoothly. The decision-making model and the software have been proved to operate well in practice, which has already improved the automation level of the urban rail transit monitoring system, and reduced the manual intervention effectively. It has been tested through regular exercises and drills that outputs from the EDSS system could be used to inform the decision-making process. It will get 
more applications with the constant improvement of technology and development of the decision support system in rail transit command center.

\section{References}

[1] Y. Qin, Z. Wang and L.M. Jia, Research on the System Framework and Application of Railway Transportation Emergency Management, China Safety Science Journal, Vol. 17, No, 1, 2007, 57-65.

[2] Q. Wang, Y.C. Huang, Decision Support Technology Research of Emergency Disposal, American Journal of Industrial and Business Management, 2013, 3, 740-745

[3] Y. P. Cui, Z. M. Tang and X. Wu, System Research Emergency Handling of Metro Accidents Based on Multi-Agent, Journal of the China Railway Society, Vol.26, No. 3, 2004, 8-12.

[4] Z. Q. Wang, M. Zhang and H. Xu, Emergency Treatment Assistant Decision System of Urban Rail Transit, International Conference on Transportation Engineering, Vol. 14, 2007, 3707-3712.

[5] Digital Plan Template and Template Digital Alarm Information Report, Beijing Jiaotong University, Beijing, 2012.

[6] Bin F, Decision Support System (DSS)-Form, Development and Future, Education Technology and Computer Science, 2009, 1002-1006. 